\title{
Through the Perilous Fight: A Case Analysis of Professional Wrestling During the COVID-19 Pandemic
}

\author{
Nicholas P. Davidson, James Du, and Michael D. Giardina \\ Florida State University
}

\begin{abstract}
The rapidly escalating COVID-19 pandemic has forced the sport industry into unchartered territory. Beginning on March 11, 2020, when the National Basketball Association suspended its season, the American sports landscape has consequently encountered an unprecedented number of temporary suspensions, postponements, and cancellations. Although most major leagues and their pertaining sports have halted to a sudden stop, professional wrestling has surprisingly continued on, including World Wrestling Entertainment's WrestleMania 36, which was held without fans in attendance. The maintenance of professional wrestling during the COVID-19 crisis has presented a unique situation, in which fans and companies involved in the sport have rallied on social media platforms behind the sport's relative normality in a time of global uncertainty. Leveraging publicly trackable Twitter data, we analyzed public sentiments toward two of the largest companies (e.g., World Wrestling Entertainment and All Elite Wrestling) in the professional wrestling industry and related trends during the widespread onset of the COVID-19 pandemic in the United States. The results represent exploratory insights surrounding the continuation of professional wrestling during the COVID-19 pandemic.
\end{abstract}

Keywords: sentiment analysis, text mining, trend analysis

The rapidly escalating COVID-19 pandemic has forced the sport industry into uncharted territory. Beginning on March 11, 2020, the sports landscape has encountered an unprecedented number of temporary suspensions, postponements, and cancellations (Aschburner, 2020). Although most major leagues and their pertaining sports have halted to a sudden stop, American professional wrestling leaders World Wrestling Entertainment (WWE) and All Elite Wrestling (AEW) continue to air new weekly in-ring programming. The maintenance of professional wrestling during the COVID-19 crisis has presented a unique situation, where sport content continues to be produced, while other sporting leagues in a vast majority have paused production. Congruently, the aforementioned major American

The authors are with the Dept. of Sport Management, Florida State University, Tallahassee, FL, USA. Davidson (ndavidson@fsu.edu) is corresponding author. 
companies involved in the sport have continued to engage with consumers via social media platforms during the sport's relative normality in a time of global uncertainty. From anticipation surrounding upcoming events to controversies over organizational firings, conversation about the sport has continued and produced multiple potential points for inquiry.

To respond, in this study, we analyzed publicly available Twitter data from both WWE's and AEW's official Twitter accounts in order to provide exploratory insights into how the sport of professional wrestling continues to be engaged with by fans during the COVID-19 pandemic, as well as the emotional responses of fans toward the sport amidst the continuation of professional wrestling during the initial outbreak of the COVID-19 pandemic in the United States.

\section{Methodology}

We began collecting data from the official Twitter accounts of WWE (@WWE) and AEW (@AEWrestling) from March 11 until April 16, 2020. Two milestone events helped us form the boundary of the timeline. On March 11, the National Basketball Association suspended gameplay indefinitely on the heels of Utah Jazz players Rudy Gobert and Donovan Mitchell testing positive for COVID-19 (Aschburner, 2020). On April 16, the White House and the Centers for Disease Control and Prevention announced the first in a series of Guidelines for Opening Up America Again, which, while not an indicator of the conclusion of the pandemic, signified a political shift toward attempting to adjust to an environment of (somewhat) open operation.

Leveraging both Twitter streaming API with Tweepy and Octoparse 8.1, we scraped 65,196 lines of tweets from the two aforementioned Twitter accounts. Both accounts and their pertaining interactions were English language centric. We additionally conducted a standardized data preprocessing procedure in order to form a clear corpus. This procedure removed irrelevant information, such as @ mention symbols, hyperlinks, and retweets. We also eliminated extra white spaces, transformed text to lower cases, deleted punctuation, and ran a stemming and lemmatization algorithm to restore words to their root forms. Via preprocessing cleansing procedures, common English stop words were removed from posts, such as "will" and "now." With each line of raw data, we extracted the original textual content, time posted, user handle, number of views, retweets, and likes published on the companies' official Twitter accounts. Two machine-learning-based natural language processing tools were used to analyze the textual content and explore the public's sentiments toward WWE and AEW during the COVID-19 outbreak in Python (version 3.8.2; Python Software Foundation, 2020) and R (version 4.0; R Core Team, 2020). Emotional theme identification was based upon the NRC Emotion Lexicon to establish word-emotion associations (Bravo-Marquez, Frank, Pfahringer, \& Mohammad, 2019).

\section{Results}

Figure 1 presents the frequency of both the top $15(N=15)$ most appearing words and hashtags derived from a document-term matrix. These most appeared "words" and trending hashtags revealed interactions surrounding WWE's and AEW's 


\section{Top 15 most appeared "words" and trending hashtags}
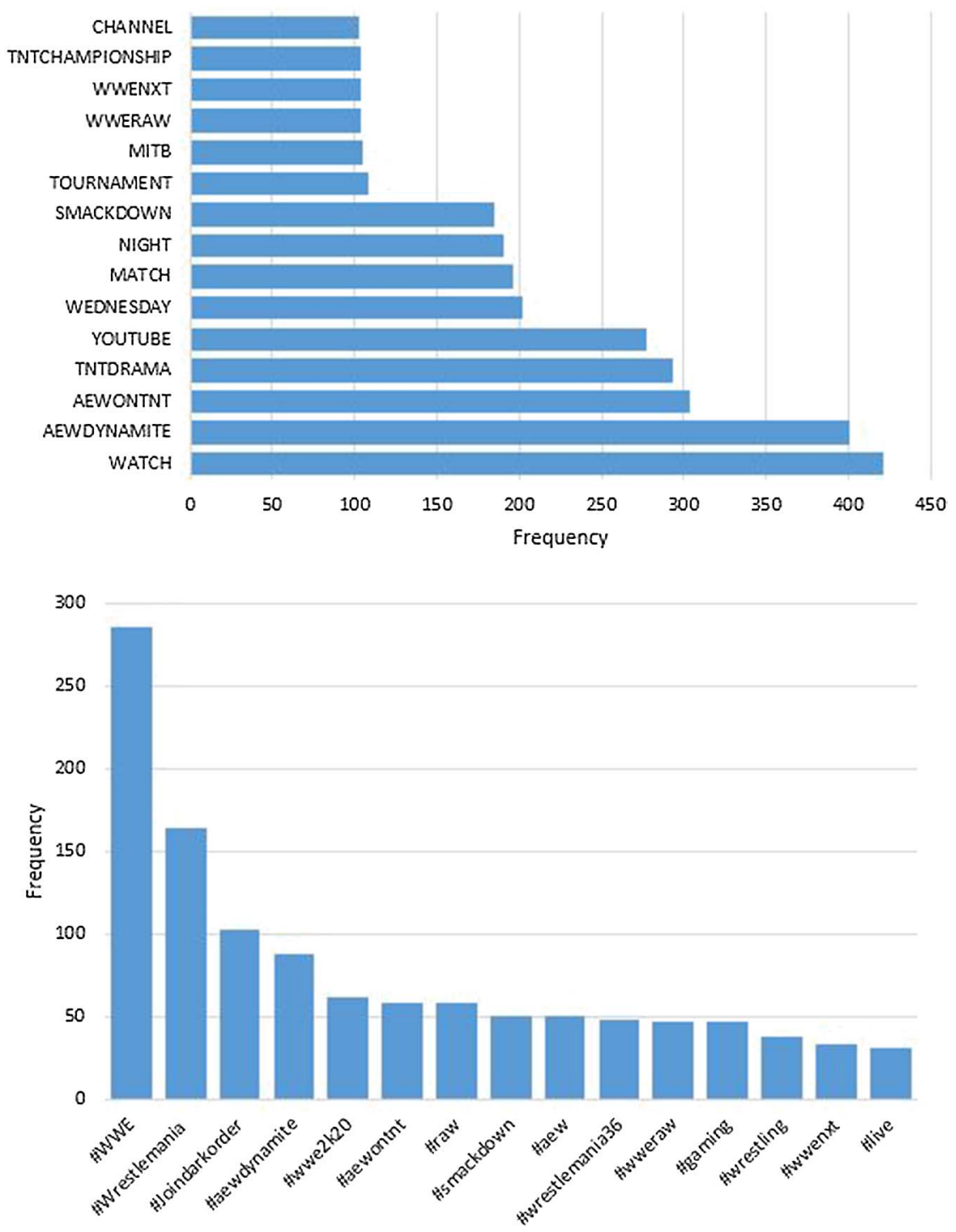

Figure 1 - Text-mining results of Twitter contents.

weekly programming. Among the top 15 most frequent words $(N=15)$, four $(n=4)$ were the names of AEW or WWE weekly programs (WWE NXT, WWE RAW, Smackdown, AEW Dynamite). Four $(n=4)$ were events either going on during or being promoted during the weekly programs (TNT Championship, 
MITB, Tournament, Match). Four $(n=4)$ were indicative of where the programming was airing (AEW on TNT, Channel, TNT Drama, YouTube). Two $(n=2)$ were representative of when the weekly programming was airing (Night, Wednesday). The one $(n=1)$ remaining "word" indicated plans or encouragement to consume weekly programming (watch). Among the top $15(N=15)$ most frequent hashtags, five $(n=5)$ were the names of weekly programs (\#AEWDynamite, \#Raw, \#Smackdown, \#WWERaw, and \#WWENXT). Three $(n=3)$ were indicative of wrestling events or storylines promoted on weekly programming (\#WrestleMania, \#JoinDarkOrder, and \#WrestleMania36). Three $(n=3)$ were indicative of watching professional wrestling (\#AEWonTNT, \#Wrestling, and \#Live). Two $(n=2)$ were the names of the two professional wrestling companies analyzed (\#WWE and \#AEW). The final two, perhaps unique from the others, were indicative of conversations surrounding WWE's latest video game release (\#WWE2k20 and \#Gaming).

Figure 2 displays the results of a sentiment analysis, measuring the extent to which the eight emotional themes (i.e., anger, anticipation, disgust, fear, joy, sadness, surprise, and trust) were embedded in the scraped Twitter data. The emotions of "anticipation," "trust," "fear," and "joy" were recognized as the four most common sentiments that resonated with the interacting fan community during the outbreak. While the absence of live audiences has placed a constraint on fans' ability to fully invest themselves in the sport, the results showed that the outbreak had not lessened the appreciation people have for professional wrestling as a spectator sport. During a time of global uncertainty, professional wrestlers within WWE and AEW who continue to perform on nationally televised programming have undertaken increased personal risk. The risks that these individuals have taken for the sake of maintaining a paycheck by providing viewers with entertainment have engendered personal feelings of concern among Twitter users interacting with the two companies. Yet, amidst the feelings of concern, Twitter users have also expressed feelings of admiration for the efforts of both companies in trying to uphold some level of normalcy in their audience's lives. Tuning into WWE's and AEW's shows during the pandemic has provided, to some degree,

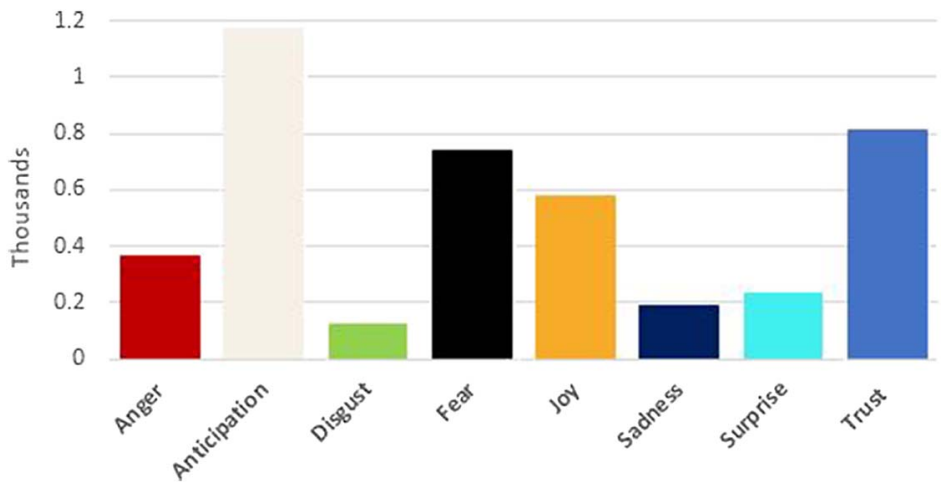

Figure 2 - Decomposition of sentiment analysis by emotions. 
a weekly alternative to the contemporary world, despite the shows' taking place in empty venues.

Using a compound sentiment score ranging from -5 to 5 , we then specified three regression models for the main time series analysis using Stata (version 16; StataCorp, 2019). To interpret the coding, -5 indicates an extremely negative sentiment, while 5 implies an extremely positive sentiment. The goal was to provide additional insights into the changes in fans' overall sentiments toward the two observed professional wrestling companies during the COVID-19 pandemic, with an emphasis on what drove the changes in the emotional responses among the interacting professional wrestling fan community. The dependent variable was an aggregated compound sentiment score at a specific date derived from the sentiment analysis. Five natural log-transformed independent variables (e.g., number of retweets, likes, and views, as well as a total quantity of positive and negative sentiments on a given day) and a variable of "trend" were included in a full-specified model, shown in Table 1.

The results of the time series analysis suggested that the number of likes $\left(\beta_{\text {likes }}=\right.$ $10.51, p<.001)$ combined with total number of positive sentiments $\left(\beta_{\text {positive }}=\right.$ $35.71, p=.036)$ were driving an upward trend ( $\left.\beta_{\text {Trend }}=0.48, p=.595\right)$ in fans' overall sentiments $\left(R^{2}=81.5 \%\right)$ toward the two leagues during the pandemic. While the diffusion of sentiments was associated with people's favored endorsement (e.g., number of likes) over time, it was not related to the propagation of their social network (e.g., number of retweets). The finding of an overall upward trend in fans' sentiments aligned with the graphical trajectory is shown in Figure 3. While fans' overall sentiment was positive in responding to the contents posted on the companies' official Twitter accounts, a symmetric pattern between the sharing of positive and negative sentiments by fans was also captured.

\section{Table 1 The Results of Time-Series Regressions}

\begin{tabular}{lccc}
\hline $\begin{array}{l}\text { Compound } \\
\text { sentiment score }\end{array}$ & $\begin{array}{c}\text { Model 1: } \\
\text { Robust } \\
\text { pooled OLS }\end{array}$ & $\begin{array}{c}\text { Model 2: } \\
\text { Time series } \\
\text { with robust SE }\end{array}$ & $\begin{array}{c}\text { Model 3: } \\
\text { Time series } \\
\text { with Newey-West SE }\end{array}$ \\
\hline Ln (no. retweet) & $1.555(1.227)$ & $1.557(1.237)$ & $1.557(1.384)$ \\
Ln (no.likes) & $10.28^{* * *}(2.562)$ & $10.51^{* * *(2.826)}$ & $10.51^{* * *}(2.701)$ \\
Ln (no. views) & $-50.45^{*}(21.09)$ & $-33.85(32.10)$ & $-33.85(31.49)$ \\
Ln (no. of positive) & $45.11^{* *}(12.77)$ & $35.71^{*}(16.19)$ & $35.71 * *(12.63)$ \\
Ln (no. of negative) & $1.272(21.26)$ & $-3.147(23.01)$ & $-3.147(27.33)$ \\
Trend & & $0.48(0.51)$ & $0.48(0.46)$ \\
$R^{2}$ & .814 & .815 & N/A \\
AIC & 276.1 & 274.3 & N/A \\
BIC & 284.9 & 281.6 & N/A \\
$F$ & 26.36 & 26.99 & 28.38 \\
Trend effects & & Yes & Yes \\
\hline
\end{tabular}

Note . AIC $=$ Akaike information criterion; $\mathrm{BIC}=$ Bayesian information criterion; OLS $=$ ordinary least-squares regression; robust standard errors in parentheses; $\mathrm{SE}=$ standard errors.

$* p<.05 . * * p<.01 . * * * p<.001$. 


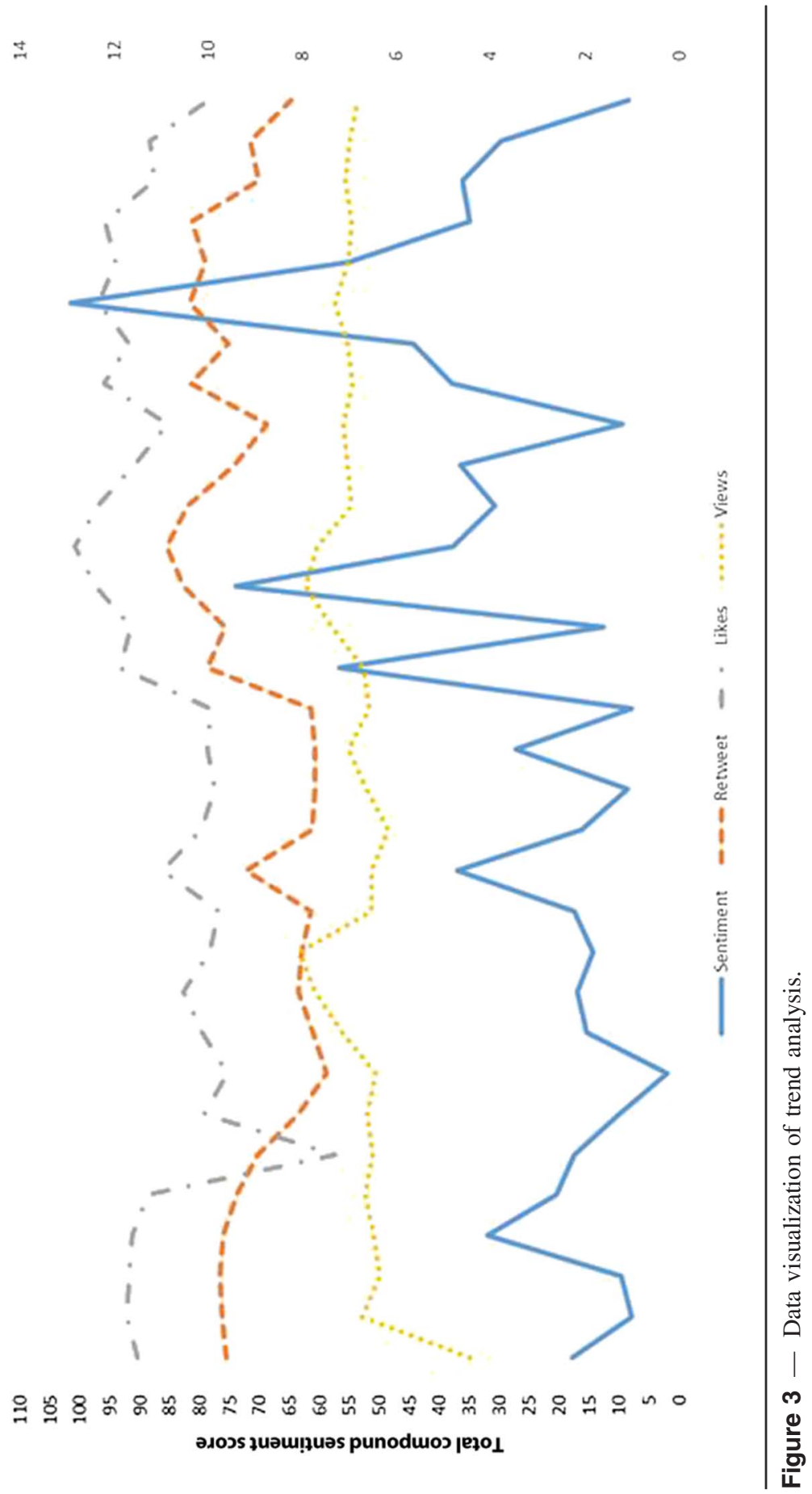




\section{Discussion and Implications}

This mixed emotional response has been well manifested in the reality of professional wrestling during the outbreak. While the production of major professional wrestling has continued in an amended fashion, the economic implications of COVID-19 on the professional wrestling industry as a whole have been felt across numerous sectors. From lost revenues due to moved or cancelled shows to the firing and furloughing of talents and the production staff, professional wrestling has entered a period of economic insecurity over the course of the pandemic.

On one hand, professional wrestlers and companies who are not the beneficiaries of large production value and television deals, also known as "independent" wrestlers or companies, have faced hardship due to their inability to promote live events that are relied on for income (Bixenspan, 2020; Barrasso, 2020a). In an effort to relieve these financial uncertainties brought on by COVID-19, independent company promoters and wrestlers have hosted empty venue wrestling shows in gyms and bars, available to watch live via online stream (Barrasso, 2020b; Blanchard, 2020). During these shows, viewers can electronically make donations that go directly to the athletes performing. The willingness of professional wrestling fans to donate their own money during a period of uncertainty to help those who entertain them, as illustrated by these events, presents an example of the supportive culture of the professional wrestling community.

On the other hand, professional wrestlers' having career and financial security concerns has not been exclusive to the independent professional wrestling scene. As shown in the trajectory (Figure 3), on April 15, WWE announced that it had come to terms with the release of several contracted talents and producers (World Wrestling Entertainment, 2020). This decision came just a week after WWE was identified as "essential business" by the state of Florida to ensure the continued production of their shows. These releases were met with a great deal of criticism among the professional wrestling community and were referred to by some as being unnecessary and lacking morally sound decision making (Thurston, 2020).

Taken together, the results of the sentiment analysis combined with the trend analysis indicated that fans of professional wrestling continued to engage with the product on a social media platform during the COVID-19 pandemic. Despite a television rating decline for both WWE and AEW during the pandemic (Konuwa, 2020), those who had engaged with the product at a high level (i.e., engaging in conversation about the sport on Twitter) continued to do so by demonstrating their interest in weekly programming via interactions with AEW and WWE Twitter posts. Consumers expressed interest in the programming by engaging in interactions both leading up to, during, and after the events. These interactions provided insight into the consumer base of a sport that, unlike its North American peers, continued production during the midst of a global crisis. This is an implication to sport scholars and practitioners alike in the sense that it demonstrates that consumers of sport, albeit in a reduced context, as indicative by rating declines, will continue to devote appreciation toward the sport they consume during a period of uncertainty.

The decomposition of the sentiment analysis by emotions indicating feelings of primarily anticipation, fear, joy, trust, and anger was indicative of feelings of both appreciation and concern. This draws implications that, while consumers 
continue to interact with the products, their feelings on the continued production and how it is being handled may not be widely polarized toward either the positive or the negative, but rather a mixture of the two. This presents a unique takeaway in that these sentiments demonstrating both positive and negative reactions can be interpreted as a reflection of the uncertain feelings surrounding society during the COVID-19 pandemic.

\section{Future Inquiry and Conclusion}

Witnessing the response of fans who are willing to support many of these athletes through continued consumption, interaction, and financial contributions during this time points to themes of genuine concern for the longevity of these individuals as not just athletes, but as human beings with livelihoods beyond their role performing an in-ring persona. This concern can be representative of a feeling of connection between fan and athlete. Without this proposed connection, the dynamic of fans supporting and interacting with professional wrestlers and companies during this hardship would have been nonexistent. In light of the uncertainty caused by the COVID-19 pandemic, social marketing programs on various social media platforms designed to maintain and enhance fan engagement should be of tremendous interest to professional wrestling organizations. The WWE and AEW during the COVID-19 pandemic have been able to maintain a consistent social media presence by tying their television content to social media content and vice versa, as evident by fan Twitter discussions often being centered around the two companies' television programming. Future studies may inquire into the sentiments of consumers on other platforms to examine the impact of COVID-19 on the professional wrestling fan community. Additionally, although the issues associated with the independent professional wrestling community during COVID-19 have been made note of, further sentiment analysis specific to independent professional wrestling may reveal different themes than those associated with major professional wrestling. Overall, while the application of this study is centered around the use of sentiment analysis, future studies could instead conduct semantic analysis in order to yield more in-depth insights into fan reactions to sport.

In conclusion, the focus of this study was built on an understanding of the sentiments and related trends facing two large professional wrestling companies surrounding the challenges associated with the COVID-19 pandemic. To put our findings in context, the exploratory nature of this study as a brief snapshot into a contemporary issue should not be representative of the long-term sentiments of fans of professional wrestling, nor is it representative of professional wrestling fans as a whole; rather, it is a snapshot of the sentiments of those who are engaging with the sport at a high level (i.e., interacting on Twitter) in the midst of global uncertainty.

\section{References}

Aschburner, S. (2020, March 12). Coronavirus pandemic causes NBA to suspend season after player tests positive. NBA. Retrieved from https://www.nba.com/article/2020/03/ 11/coronavirus-pandemic-causes-nba-suspend-season 
Barrasso, J. (2020a, March 19). GCW show raising money for wrestlers impacted by coronavirus. Sports Illustrated. Retrieved from https://www.si.com/wrestling/2020/03/ 19/gcw-acid-cup-coronavirus-show

Barrasso, J. (2020b, April 19). Performers struggle as coronavirus shuts down indie wrestling. Sports Illustrated. Retrieved from https://www.si.com/wrestling/2020/04/ 19/independent-wrestling-coronavirus-shutdown

Bixenspan, D. (2020, March 13). A WrestleMania coronavirus shutdown would be a catastrophe for indie wrestling. Vice. Retrieved from https:/www.vice.com/en_us/ article/pkevx7/a-wrestlemania-coronavirus-shutdown-would-be-a-catastrophe-for-indiewrestling-wwe

Blanchard, M. (2020, March 17). WrestleTalk Showcase: No fans Monday a huge success thanks to Will Ospreay. The Overtimer. Retrieved from https://theovertimer. com/2020/03/wrestletalk-showcase-no-fans-monday-a-huge-success-thanks-to-willospreay/

Bravo-Marquez, F., Frank, E., Pfahringer, B., \& Mohammad, S.M. (2019). AffectiveTweets: A Weka package for analyzing affect in tweets. Journal of Machine Learning Research, 20(92), 1-6. Retrieved from http://jmlr.org/papers/v20/18-450.html

Konuwa, A. (2020, April 2). AEW, WWE NXT viewership down double digits as both desperately seek workarounds amid COVID-19 pandemic. Forbes. Retrieved from https:// www.forbes.com/sites/alfredkonuwa/2020/04/02/aew-wwe-nxt-down-double-digitsas-both-desperately-seek-workarounds-amid-covid-19-pandemic/\#645b745d19c3

Python Software Foundation. (2020). Python Language Reference, version 3.8.2.. Retrieved from http://www.python.org

R Core Team. (2020). R: A language and environment for statistical computing. R Foundation for Statistical Computing, Vienna, Austria.. Retrieved from https:// www.R-project.org/

StataCorp. (2019). Stata Statistical Software: Release 16. College Station, TX: StataCorp LLC.

Thurston, B. (2020, April 20). WWE layoffs were morally heartless and economically unnecessary. Wrestlenomics. Retrieved from https://wrestlenomics.com/2020/04/20/ wwe-layoffs-were-morally-heartless-and-economically-unnecessary/

World Wrestling Entertainment. (2020, April 15). Rusev, Kurt Angle and other superstars released. WWE. Retrieved from https://www.wwe.com/article/wwe-talent-releaseskurt-angle-drake-maverick-karl-anderson 